\title{
Optical gain in NV- colour centres for highly-sensitive magnetometry: a theoretical study
}

\author{
Vasili G. Savitski \\ Institute of Photonics, Dept. of Physics, University of Strathclyde, Glasgow, G1 1RD, UK \\ vasili.savitski@strath.ac.uk
}

\begin{abstract}
Optical gain in an amplifier based on NV- colour centres (CC) in diamond is proposed as a new method for optical magnetic field detection. An analytical expression for magnetic field sensitivity with this method is deduced. The sensitivity is highest at the pump intensities about 100 times above the saturation intensity of NV- CC and can reach values of $\sim 1.4 \mathrm{fT} / \sqrt{ } \mathrm{Hz}$ in a pump-probe configuration with $\mathrm{NV}$ - CC concentration in diamond of $2.5 \mathrm{ppm}$. Collection efficiency of probe emission can be simpler and significantly higher than that of NV-CC luminescence, used for conventional optical magnetometry in diamond. This makes this method attractive for applications requiring remote magnetic field sensing.
\end{abstract}

\section{Introduction}

Highly-sensitive optically pumped magnetometers operating at room temperatures are of interest as alternative to cryogenic-temperature operated systems like superconducting quantum interference device sensors [1]. Among them magnetometers based on NV-colour centres (CC) in diamond have attracted considerable attention for magnetic field sensing in biology and with nanometer spatial resolution [2], with the best sensors having sub-pT magnetic field sensitivity [3]. These devices can be used in a number of applications including magneto cardiology and magnetoencephalography $[4,5]$.

One of the fundamental limitations to the sensitivity of NV- CC magnetometers is in the number of detectable photons [6]. This number is limited by the collection efficiency of the optics, the number of $\mathrm{NV}$ - defects in the diamond and the effect of total internal reflection [7]. Recently, some theoretical studies predicted the potential for using laser threshold in NV- for magnetometry to achieve fT/ $\sqrt{\mathrm{Hz}}$ sensitivity $[8,9]$. Laser action in NV- CC is, however, yet to be demonstrated. In this paper, the prospects for instead using optical gain in NV- CC for highly-sensitive magnetometry are analysed theoretically.

The simplified electronic level scheme of NV- CC in diamond, similar to the one presented in [8,9], is shown in Fig. 1. The CC ground state is a spin-triplet with ${ }^{3} \mathrm{~A}_{2}$ symmetry [10]. Three spin-projection states $m_{s}=0$ (level 1 in Fig. 1 ) and $m_{s}= \pm 1$ (shown as a single level (level 4 ) in Fig. 1 for simplicity) have a splitting of $v_{0} \sim 2.88 \mathrm{GHz}$ at zero magnetic field [11]. The basic principle of a magnetometer based on $\mathrm{NV}-\mathrm{CC}$ in diamond is as follows [10]: at room temperature the population of the ground state spin levels $\mathrm{m}_{\mathrm{s}}=0$ and $\mathrm{m}_{\mathrm{s}}= \pm 1$ is almost equal. Optical excitation results in spin polarisation: $\sim 80 \%$ of the total 


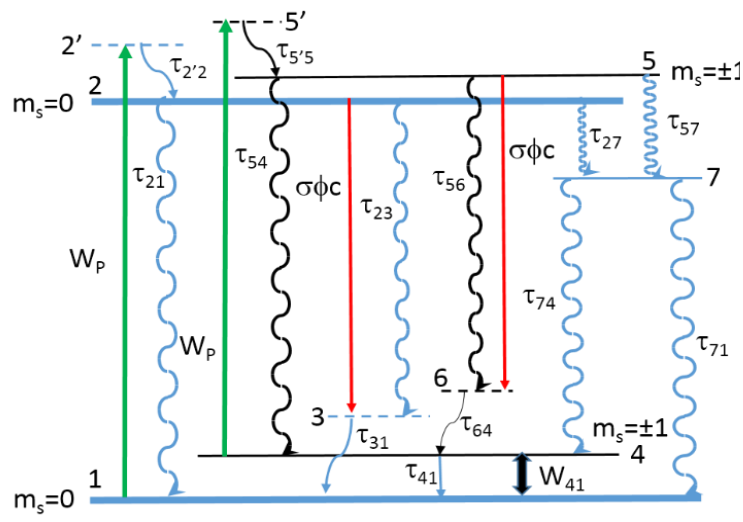

Fig. 1. Simplified electronic level scheme of NV- CC in diamond describing optical gain detected magnetic resonance technique. Levels 1 and 2 are the ground and excited spin-projection states with $m_{s}=0$. Levels 4 and 5 are the ground and excited spin-projection states with $\mathrm{m}_{\mathrm{s}}= \pm 1$. Levels 3 and 6 are the ground state vibrational states. Levels 2' and 5' are excited state vibrational states. Level 7 is a simplified representation of a singlet system ${ }^{1} \mathrm{~A}_{1}+{ }^{1} \mathrm{E}$.

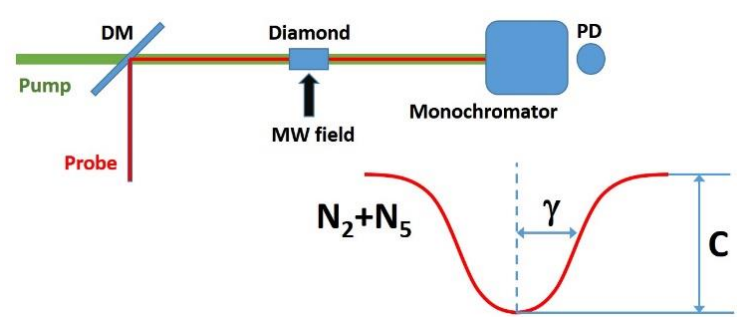

Fig. 2. Schematic of a pump-probe setup for OGDMR measurements. DM is a dichroic mirror (high reflectivity at the probe wavelength and high transmittance at the pump wavelength), PD is a photodetector. The curve $\mathrm{N}_{2}+\mathrm{N}_{5}$ represents schematically the change in population of the levels $\mathrm{N}_{2}$ and $\mathrm{N}_{5}$ as a function of frequency of applied micro-wave (MW) field in the presence of both pump and probe emission.

population is in the $m_{s}=0$ ground state [12]. It means that any microwave (MW) field resonant with the ground state transition $\mathrm{m}_{\mathrm{s}}=0 \rightarrow \mathrm{m}_{\mathrm{s}}= \pm 1$ can drive some population from the ground $\mathrm{m}_{\mathrm{s}}=0$ to the ground $\mathrm{m}_{\mathrm{s}}= \pm 1$. Intensity of any spontaneous emission taken place between levels

2 (5) and 1 (4), measured as a function of the MW field frequency will have a local minimum at 2.88 $\mathrm{GHz}$ resonant frequency (the so-called optically detected magnetic resonance (ODMR)). Any magnetic field applied in this case will effectively "move" spin levels $m_{s}=0$ and $m_{s}= \pm 1$ away from the resonant frequency, which will manifest itself in increase of intensity of spontaneous emission detected at MW frequency of $2.88 \mathrm{GHz}$.

\section{Optical gain detected magnetic resonance}

"Conventional" magnetometers based on NV- CC relate the change in the intensity of spontaneous emission (luminescence) to the magnetic field strength via the ODMR technique [10]. An alternative route for measuring the magnetic field strength, suggested in the present paper, is based on detecting the intensity of the external (probe) light, resonant with the optical frequency between levels 1 (4) and 2 (5), which can be amplified via the stimulated emission process between these levels. This optical gain detected magnetic resonance (OGDMR) technique can be realised using a pump-probe technique (see Fig. 2): the pump and the probe beams are combined at a dichroic mirror and then are both focused on the diamond sample. The probe beam is then spectrally filtered using a monochromator and any change in its intensity caused by the optical gain, can be detected using a photodetector (Fig. 2).

The most efficient optical amplification of probe emission from e.g. a single-mode laser diode, when the diamond is pumped at e.g. $532 \mathrm{~nm}$, can be observed in the spectral region $\sim 670-800 \mathrm{~nm}$ [13]. This spectral range corresponds to induced transitions between the excited spin levels $\mathrm{m}_{\mathrm{s}}=0$ (level 2) or $\mathrm{m}_{\mathrm{s}}= \pm 1$ (level 5) and corresponding ground vibrational states labelled as 3 and 6 in Fig. 1. The rate equations describing the populations $N$ of the various levels shown in Fig. 1 are given below, where the following had been assumed: $\mathrm{N}_{2}=\mathrm{N}_{5}=\mathrm{N}_{3}=\mathrm{N}_{6}=0, \tau_{23}=\tau_{56} \rightarrow \infty$ and $\tau_{21}=\tau_{54}=\tau_{\text {rad }} \eta$ (where $\tau_{\text {rad }}$ is the radiative lifetime of $\mathrm{NV}-\mathrm{CC}$ and $\eta$ is the luminescence quantum yield), $\tau_{57}<<\tau_{27}$ [14] and $\tau_{2}{ }^{\prime}=\tau_{5}{ }^{\prime}=\tau_{31}=\tau_{64} \rightarrow 0$ :

$$
\frac{\partial N_{1}}{\partial t}=-N_{1}\left(1 / 4 W_{41}(\delta)+W_{P}\right)+N_{2}\left(\sigma \phi c+\frac{1}{\tau_{21}}\right)+N_{4}\left(1 / 4 W_{41}(\delta)+\frac{1}{\tau_{41}}\right)+\frac{N_{7}}{\tau_{71}}
$$




$$
\begin{gathered}
\frac{\partial N_{2}}{\partial t}=W_{P} N_{1}-N_{2}\left(\sigma \phi c+\frac{1}{\tau_{21}}\right) \\
\frac{\partial N_{4}}{\partial t}=1 / 4 N_{1} W_{41}(\delta)-N_{4}\left(1 / 4 W_{41}(\delta)+W_{p}+\frac{1}{\tau_{41}}\right)+N_{5}\left(\sigma \phi c+\frac{1}{\tau_{54}}\right)+\frac{N_{7}}{\tau_{74}} \\
\frac{\partial N_{5}}{\partial t}=W_{P} N_{4}-N_{5}\left(\sigma \phi c+\frac{1}{\tau_{54}}+\frac{1}{\tau_{57}}\right) \\
\frac{\partial N_{7}}{\partial t}=\frac{N_{5}}{\tau_{57}}-N_{7}\left(\frac{1}{\tau_{71}}+\frac{1}{\tau_{74}}\right) \\
N_{\text {total }}=N_{1}+N_{2}+N_{4}+N_{5}+N_{7}
\end{gathered}
$$

where $\tau_{x y}$ represents relaxation time of the corresponding transition $x \rightarrow y, \mathrm{~W}_{\mathrm{p}}$ is the pumping rate, $\mathrm{W}_{\mathrm{P}}=$ $\frac{\mathrm{P}_{\text {pump }}}{\mathrm{h} v_{\text {pump }} \mathrm{V}_{\text {pump }} \mathrm{N}_{\text {total }}}\left(\mathrm{P}_{\text {pump }}\right.$ is the absorbed pump power, $\mathrm{h} v_{\text {pump }}$ is the pump photon energy, $\mathrm{V}_{\text {pump }}$ is the pumping volume and $\mathrm{N}_{\text {total }}$ is the concentration of $\mathrm{NV}-\mathrm{CC}$ ). $\mathrm{W}_{41}$ is the stimulated transition probability between the spin levels of the ground state, $\mathrm{W}_{41}(\delta)=\frac{\omega_{\mathrm{R}}^{2}}{\Delta \omega_{\mathrm{A}}}\left[\frac{\left(\omega_{\mathrm{A}} / 2\right)^{2}}{\delta^{2}+\left(\omega_{\mathrm{A}} / 2\right)^{2}}\right]\left(\omega_{\mathrm{R}}\right.$ is Rabi frequency, which is proportional to square root of the applied MW power, $\Delta \omega_{A}=1 / \tau_{41}+2 / T_{2}$ [15], with $\tau_{41}$ being the spin energy relaxation time (longitudinal relaxation time) and $\mathrm{T}_{2}$ is the spin dephasing time, $\delta= \pm B \gamma_{e} / 2 \pi$ is the microwave detuning from the central frequency $v_{0}(B$ is the projection of the measured magnetic field along the $\left\langle 111>\right.$ axis of NV- CC, $\gamma_{e}=1.761 \times 10^{11} \mathrm{~Hz} / \mathrm{T}$ is the electron gyromagnetic ratio)) in approximation that $\mathrm{W}_{41}<<\Delta \omega_{\mathrm{A}}$ [15]. In a diamond with an NV- CC concentration of $2.5 \mathrm{ppm}$ (considered below in calculations), $\tau_{41}$ is $\sim 3 \mathrm{~ms}$ and $\mathrm{T}_{2}$ is $\sim 0.2 \mu \mathrm{s}$ [16, 17], and therefore the approximation that $\mathrm{W}_{41}<<\Delta \omega_{\mathrm{A}}$ is satisfied at $\omega_{\mathrm{R}}<10 \mathrm{MHz} . \sigma$ is the emission cross section of NV- CC. $\phi$ is the photon density of the external (probe) light source, $\phi=\frac{\mathrm{P}_{\text {probe }}}{\mathrm{h} v_{\text {probe }} \mathrm{cA}}\left(\mathrm{P}_{\text {probe }}\right.$ is the probe power, $\mathrm{h} v_{\text {probe }}$ is the probe photon energy, $\mathrm{c}$ is the speed of light, $\mathrm{A}$ is the cross-sectional area of the probe beam on the sample). The factor $1 / 4$ is included into Eq. (1) to account for the fact that only a quarter of the total NV-population will be oriented along the direction of the magnetic field and be in resonance with the MW field, as set out in [18].

Taking into account that $\tau_{74} \sim 0.8 \tau_{71}[12,14]$, and $\tau_{54}=\tau_{21}$, the solution for $\mathrm{N}_{2}$ and $\mathrm{N}_{5}$ in the stationary case can be written as:

where

$$
N_{2}=\frac{N_{2 \max } W_{P} N_{\text {total }}}{W_{p} N_{\text {total }}+N_{2 M W} N_{2 \max }}
$$

$$
\begin{gathered}
N_{2 \max }=\frac{W_{P} N_{\text {total }}}{\Phi+W_{p}} \\
N_{2 M W}=\frac{W_{41}(\delta) W_{P} \Phi\left[\frac{\Phi+1 / \tau_{57}}{W_{P}}+\tau_{71} / 2.25 \tau_{57}+1\right]}{\left(W_{P}+W_{41}(\delta)+1 / \tau_{41}\right)\left(\Phi+1 / \tau_{57}\right)-W_{P}\left(\Phi+1 / 1.8 \tau_{57}\right)} \\
\Phi=\sigma \phi c+1 / \tau_{21} \\
N_{5}=\frac{N_{2 M W} N_{\text {total }}}{\left[\Phi+W_{P}+N_{2 M W}\right]\left[\frac{\Phi+1 / \tau_{57}}{W_{P}}+\tau_{71} / 2.25 \tau_{57}+1\right]}
\end{gathered}
$$

The relaxation times are: $\tau_{57}=18 \mathrm{~ns}$ (estimated from the lifetime of the excited spin-projection state with $\mathrm{m}_{\mathrm{s}}= \pm 1$ (level 5) of $\sim 7.5 \mathrm{~ns}$ [12] and the luminescence lifetime $\tau_{54}=13 \mathrm{~ns}$ ): $1 / \tau_{5}=1 / \tau_{57}+1 / \tau_{54} . \tau_{71}=400 \mathrm{~ns}$ (estimated from the lifetime of the singlet level 7 of $\sim 178 \mathrm{~ns}$ and the relation $\tau_{74}=0.8 \tau_{71}$ [14]): $1 / \tau_{7}=1 / \tau_{71}+1 / \tau_{74}$. The radiative lifetime of NV- CC is $\tau_{\text {rad }}=13 \mathrm{~ns}$ [19] and the reported values of the luminescence quantum yield range between $100 \%$ [19] and 60\% [13].

In the absence of any MW field resonant with the energy spacing between the ground spin levels $\mathrm{m}_{\mathrm{s}}=0$ and $\mathrm{m}_{\mathrm{s}}= \pm 1$ (i.e. at $\mathrm{W}_{41}=0$ ), optical excitation between the ground states and the corresponding vibrational states (levels 2 ' and $5^{\prime}$ ') will result in $\sim 80 \%$ of the total population to be accumulated in the 
ground $\mathrm{m}_{\mathrm{s}}=0$ state (level 1) [12], with $\mathrm{N}_{2}$ reaching its maximum value $\left(\mathrm{N}_{2 \max }\right)$ while $\mathrm{N}_{5}=0$ at $\mathrm{W}_{41}=0$ (see Eqs. (2)-(4) and (6)). This happens because of the high probability for population in the excited $\mathrm{m}_{\mathrm{s}}= \pm 1$ levels to decay to the ground level $\mathrm{m}_{\mathrm{s}}=0$ via the singlet level $7\left(\tau_{57}<<\tau_{27}\right.$ [14]). Population in the excited spin level $\mathrm{m}_{\mathrm{s}}=0\left(\mathrm{~N}_{2}\right)$ decays either spontaneously (luminescence, transition $2 \rightarrow 1$ ) or by the stimulated process $(\sigma \phi c)$ via the transition induced by the probe light, to one of the vibrational states of the CC (transition $2 \rightarrow 3$ ). The vibrational states then decay very rapidly back to the ground state $m_{s}=0$. The probe emission will experience maximum gain due to the stimulated emission process.

When, in the presence of the pump, the MW field is applied to the system with a frequency of $v_{0} \sim 2.88$ $\mathrm{GHz}$, the ground spin level $\mathrm{m}_{\mathrm{s}}=0$ starts to be depopulated due to the MW field induced transitions to the ground level spin $m_{s}= \pm 1$ (level 4). From the ground $m_{s}= \pm 1$ level the population is transferred via the excited $m_{s}= \pm 1$ state to singlet long lived level 7. In that case the excited spin level with $m_{s}=0$ (level 2 ) will experience some depopulation, while the population of the excited spin level with $m_{s}= \pm 1$ (level 5) will start to increase. The total population $N_{2}+N_{5}$ will, however, be lower than $N_{2 \max }$. This will result in less optical gain for the probe light at the resonant MW frequency (see Fig. 2, curve $\left(\mathrm{N}_{2}+\mathrm{N}_{5}\right)$ ).

With both the optical pump and MW field present, any magnetic field under investigation will lift the degeneracy, and hence cause splitting, of the ground spin level $\mathrm{m}_{\mathrm{s}}= \pm 1$ (level 4). This can be thought of as shifting level 4 away from the MW resonance of $\sim 2.88 \mathrm{GHz}$. In this case, microwave-driven depopulation of the ground state $\mathrm{m}_{\mathrm{s}}=0$ level (level 1) will be less efficient, and as a result the excited state $m_{s}=0$ level (level 2) will be populated more rapidly by optical pumping than in the case where only the MW field is applied. As a result, the probe emission will experience more gain. The value of the applied magnetic field is related to the change in the optical gain for the probe light.

\section{The minimum detectable magnetic field under OGDMR}

The power of the probe beam after the sample $\left(P_{\text {out }}\right)$, assuming that the intensity of the probe is much less than the saturation intensity of the NV- CC $\left(\sim 0.6 \mathrm{MW} / \mathrm{cm}^{2}\right.$ at $\left.710 \mathrm{~nm}[13]\right)$, can be written as [20]:

$$
P_{\text {out }}=P_{\text {probe }} \exp \left(\sigma L\left(N_{2}+N_{5}\right)\right)
$$

and then, similar to $[18,21]$ :

$$
P_{\text {out }}=P_{\text {probe }} \exp \left[\sigma L N_{2 \max }\left\{1-C \frac{\gamma^{2}}{\delta^{2}+\gamma^{2}}\right\}\right]
$$

where $L$ is the sample length; $C$ is the OGDMR contrast in the change of the sum of the populations of levels $N_{2}$ and $N_{5}$, driven by the MW and magnetic fields, $C=1-\frac{N_{2}(0)+N_{5}(0)}{N_{2} \max }$ (with $N_{2}(0)$ and $N_{5}(0)$ being the populations of levels 2 and 5 at the MW resonance $v_{0}$ in the absence of any external magnetic field); $\gamma$ is the HWHM linewidth of this change (see Fig. 2) and can be found by solving the equation $\frac{d^{2} N_{2 M W}}{d \delta^{2}}=0$ :

$$
\gamma=\frac{3}{8} \sqrt{\frac{\left(\Phi+1 / \tau_{57}\right) \omega_{\mathrm{R}}^{2} \Delta \omega_{\mathrm{A}}}{\left(\Phi+1 / \tau_{57}\right)\left(W_{P}+1 / \tau_{41}\right)-W_{P}\left(\Phi+1 / 1.8 \tau_{57}\right)}+\Delta \omega_{\mathrm{A}}^{2}}
$$

The minimum detectable magnetic field is given by [16]:

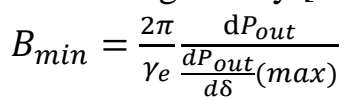

where $d P_{\text {out }}$ is the minimum detectable change in the probe power. During measurement time $t_{m}$ the number of detected photons is $P_{\text {probe }} t_{m} / h v_{\text {probe }}$, and the corresponding photon shot-noise can be expressed as $\sqrt{\frac{\text { probet }_{m}}{h v_{\text {probe }}}}[16]$.

The first derivative of Eq. (7) with respect to $\delta$ is:

$$
\frac{d P_{\text {out }}}{d \delta}=\frac{2 P_{\text {probe }} \sigma L N_{2 \max } C \gamma^{2} \delta}{\left(\delta^{2}+\gamma^{2}\right)^{2}} \exp \left[\sigma L N_{2 \max }\left\{1-C \frac{\gamma^{2}}{\delta^{2}+\gamma^{2}}\right]\right.
$$

This derivative has maximum value at $\delta \approx 0.77 \gamma[21]$ :

$$
\frac{d P_{\text {out }}}{d \delta}(\max ) \approx \frac{P_{\text {probe }} \sigma L N_{2 \max } C}{1.65 \gamma} \exp \left[\sigma L N_{2 \max }\left\{1-\frac{C}{1.6}\right\}\right] \text { (11). }
$$


By replacing $P_{\text {probe }}$ on the right-hand side of the Eq. (10) with the number of detected photons during measurement time $t_{m}$, the photon shot-noise limited magnetic field sensitivity that can be measured using optical gain of the probe emission can be expressed as:

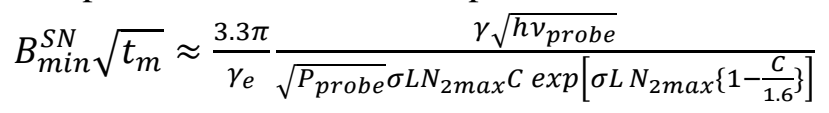

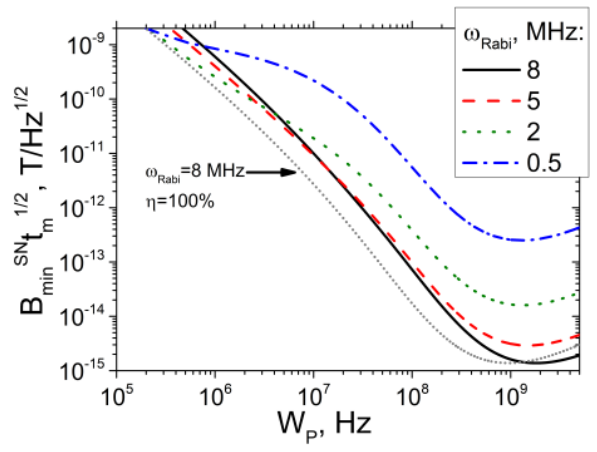

Fig. 3. Shot-noise magnetic field sensitivity using OGDMR technique, as a function of pumping rate for different Rabi frequencies.

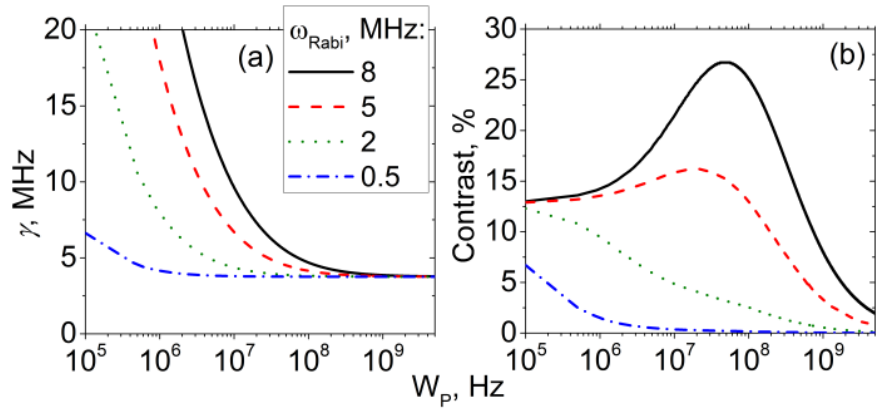

Fig. 4. HWHM linewidth $\gamma$ (a) and contrast $C$ (b) as functions of pumping rate for different Rabi frequencies using OGDMR technique.

\section{Discussion}

The major difference between the luminescence and optical gain methods for magnetic field detection is that the magnetic field sensitivity in case of optical gain detected magnetic resonance (OGDMR) is the function of probe power (eq. (12)) and does not depend on the collection efficiency of luminescence from NV-CC (as in the case of luminescence ODMR [2]). With the probe emission from a single-mode laser diodes, almost all the light transmitted through the diamond sample can be collected on the photodetectot (Fig. 2). This is because this method is less sensitive to the light-trapping effect [7], as typical divergence of the probe light from a single-mode laser diode, mentioned below, inside the diamond $\left(\sim 2^{\circ}\right)$ is lower than the critical angle of the total internal reflection $\left(\sim 24^{\circ}[22]\right)$. Besides, the light-trapping effect can be eliminated even further by using anti-reflection coating on diamond surfaces [23]. This in principle allows one to increase the shot-noise magnetic field sensitivity by simply increasing the probe power (as long as the probe intensity stays well below the saturation intensity of NV- CC) with all the rest of the pump parameters being the same. The sensitivity is, however, still a function of the contrast $C$ and linewidth $\gamma$ of OGDMR (Fig. 3). It is plotted in Fig. 3 for different Rabi frequencies as a function of the pumping rate. The calculations are for a $5 \mathrm{~mm}$ long diamond sample with an NV-CC concentration of $2.5 \mathrm{ppm}$, illuminated with $40 \mathrm{~mW}$ of probe power at the wavelength of $710 \mathrm{~nm}$ (typical output power for a single-mode laser diode at this wavelength), where the NVemission cross-section is $3.6 \times 10^{-17} \mathrm{~cm}^{2}$ [13]. The calculations are done assuming $60 \%$ luminescence quantum yield $(\eta=60 \%)$ [13].

Magnetic field sensitivity is highest at a certain optimum pumping rate for each Rabi frequency (Fig. 3 ). This is a result of a complex dependence of the maximum excited-state level population $N_{2 \max }$, the contrast $C$ and the linewidth $\gamma$ of the resonance on the pumping rate $W_{p}$. These optimum pumping rates $(\sim 2 \mathrm{GHz})$ are about 2 orders of magnitude higher than the saturation pumping rate of the optical transition 1 (4) $\rightarrow 2$ (5) for given pumping conditions and CC concentration. At this pumping rate the maximum excited-state level population starts to be saturated and is close to the total population of NV-

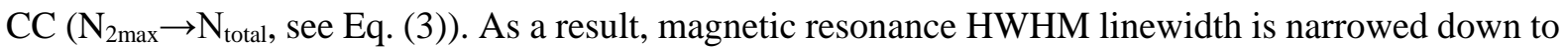
$3 \Delta \omega_{\mathrm{A}} / 8$ (see Fig. 4(a) and Eq. (8)), but the contrast is still high enough ( $5 \%$ for Rabi frequency of 8 $\mathrm{MHz}$ ) for efficient magnetic field detection (Fig. 3 (b)). With further increasing of the pumping rate 
$(\mathrm{Wp} \rightarrow \infty), \mathrm{N}_{2 \max } \rightarrow \mathrm{N}_{\text {total }}, \mathrm{C} \rightarrow 0$ and $\gamma \rightarrow 3 \Delta \omega_{\mathrm{A}} / 8$ and the minimum magnetic field sensitivity starts decreasing again (Fig. 3). Optical gain magnetic resonance linewidth narrowing, observed as the pumping rate is increased (Fig. 4 (a)) for different Rabi frequencies, is similar to the effect reported in [18] for luminescence magnetic resonance. It was attributed to the fact that the "effective" spin energy relaxation rate ("effective" longitudinal relaxation rate) is a sum of the longitudinal relaxation rate $1 / \tau_{41}$ and the pumping rate $\mathrm{W}_{\mathrm{p}}$ (see Eq. (4)) [18]. The contrast $C$ of the resonance decreases monotonically with the pumping rate up to the Rabi frequency of $\sim 5 \mathrm{MHz}$, when a local maximum in the contrast appears at a pumping rate close to the saturation pumping rate of the optical transition 2(5) $\rightarrow 3(6)$ (Fig. 3 (b)).

The minimum detectable magnetic field for the conditions described above at the optimum pumping rate of $\sim 1 \mathrm{GHz}$ is $\sim 1.4 \mathrm{fT} / \sqrt{\mathrm{Hz}}$ at a Rabi frequency of $8 \mathrm{MHz}$. This is better than the theotical shotnoise limited magnetic field sensitivity of $1.86 \mathrm{fT} / \sqrt{ } \mathrm{Hz}$ [9] in a diamond with $16 \mathrm{ppm}$ of NV-, using a laser threshold magnetometry. The approach suggested here does not require the fabrication of a laser resonator, which makes the system simpler.

Luminescence quantum yield $(\eta)$ affects the optimum pumping rate required to achieve the highest magnetic field sensitivity. The higher is the quantum yield, the lower is the optimum pumping rate, which is illustrated in Fig. 3 with a dotted grey line, which represents the OGDMR magnetic field sensitivity as a function of pumping rate for Rabi frequency of $8 \mathrm{MHz}$ and luminescence quantum yield of $100 \%$. The optimum pumping rate in this case is $\sim 1 \mathrm{GHz}$, which is 2 times lower than that with the luminescence quantum yield of $60 \%$.

The OGDMR sensitivity can be further increased at low temperatures. Order of magnitude slower spin dephasing time $T_{2}$ was reported in isotopically pure diamond [24] when the temperature was decreased from 300 down to $160 \mathrm{~K}$. Assuming similar increase in $\mathrm{T}_{2}$ at $160 \mathrm{~K}$ in a diamond sample considered in this paper, one can expect 10 times narrower linewidth $\gamma$ and order of magnitude better magnetic field sensitivity in comparison with the room temperature case considered above.

By manufacturing a waveguide in the diamond containing NV-CC detection of the amplified probe can be simplified significantly. This is due to a fact that in waveguides both pump and probe light propagate collinearly and aligned perfectly with each over, without any need for mechanical alignment of the pump-probe setup (Fig. 2). Moreover, the interaction length can be increased further, while still maintaining the small pumping area in the crystal. A diamond rib waveguide with height and width of $\sim 5 \mu \mathrm{m}$ has been suggested to be sufficient for strong mode confinement and low scattering loss [25]. Waveguiding would make the device compatible with fiber technology for pumping the diamond and collecting the amplified emission.

\section{Conclusion}

In conclusion, an optical gain detected magnetic resonance method for magnetic field measurements using NV- CC in diamonds is described theoretically. This method is based on detecting changes in optical gain of a probe beam passing through the optically pumped diamond caused by applied microwave and magnetic fields. This method does not require collection of NV-CC luminescence over a large solid angle, and the magnetic field sensitivity is instead proportional to the square root of the probe beam power. Shot-noise limited magnetic field sensitivity of $\sim 1.4 \mathrm{fT} / \sqrt{\mathrm{Hz}}$ is predicted to be possible with this method in a simple pump-probe geometry.

Funding. European Research Council (grant 278389)

Acknowledgment. The author is grateful to Alan Kemp and Łukasz Dziechciarczyk for their comments on the manuscript.

[1] Budker D, Romalis M 2007 Optical magnetometry Nat Phys 3 227-34

[2] Aharonovich I, Prawer S 2014 Quantum Information Processing with Diamond. Principles and Applications. Prawer S, Aharonovich I, editors Elsevier 
[3] Wolf T, Neumann P, Nakamura K, Sumiya H, Ohshima T, Isoya J, et al. 2015 Subpicotesla Diamond Magnetometry Physical Review X 5041001

[4] Barry J F, Turner M J, Schloss J M, Glenn D R, Song Y, Lukin M D, et al. 2016 Optical magnetic detection of single-neuron action potentials using quantum defects in diamond Proceedings of the National Academy of Sciences of the United States of America 113 14133-8

[5] Dale M W, Morley G W Medical applications of diamond magnetometry: commercial viability arXiv:170501994

[6] Acosta V M, Bauch E, Jarmola A, Zipp L J, Ledbetter M P, Budker D 2010 Broadband magnetometry by infrared-absorption detection of nitrogen-vacancy ensembles in diamond Applied Physics Letters 97174104

[7] Le Sage D, Pham L M, Bar-Gill N, Belthangady C, Lukin M D, Yacoby A, et al. 2012 Efficient photon detection from color centers in a diamond optical waveguide Phys Rev B $\mathbf{8 5} 121202$

[8] Faraon A, Santori C M, Beausoleil R G, inventorsColor centers affected by magnetic fields to produce light based on lasing. USA patent US20140072008 A1. 2014.

[9] Jeske J, Cole J H, Greentree A D 2016 Laser threshold magnetometry New Journal of Physics 18013015

[10] Doherty M W, Manson N B, Delaney P, Hollenberg L C L 2011 The negatively charged nitrogen-vacancy centre in diamond: the electronic solution New Journal of Physics $\mathbf{1 3} 025019$

[11] Loubser J H N 1977 Optical spin-polarisation in a triplet state in irradiated and annealed type $1 \mathrm{~b}$ diamonds Diamond Research 9 11-4

[12] Doherty M W, Manson N B, Delaney P, Jelezko F, Wrachtrup J, Hollenberg L C L 2013 The nitrogen-vacancy colour centre in diamond Physics Reports 528 1-45

[13] Fraczek E, Savitski V G, Dale M, Breeze B G, Diggle P, Markham M, et al. 2017 Laser spectroscopy of NV- and NV0 colour centres in synthetic diamond Optical Materials Express 72571 85

[14] Robledo L, Bernien H, van der Sar T, Hanson R 2011 Spin dynamics in the optical cycle of single nitrogen-vacancy centres in diamond New Journal of Physics 13025013

[15] Siegman A E 1986 Lasers University Science Books

[16] Rondin L, Tetienne J P, Hingant T, Roch J F, Maletinsky P, Jacques V 2014 Magnetometry with nitrogen-vacancy defects in diamond Reports on Progress in Physics 77056503

[17] Acosta V M, Bauch E, Ledbetter M P, Santori C, Fu K M C, Barclay P E, et al. 2009 Diamonds with a high density of nitrogen-vacancy centers for magnetometry applications Phys Rev B 80115202

[18] Jensen K, Acosta V M, Jarmola A, Budker D 2013 Light narrowing of magnetic resonances in ensembles of nitrogen-vacancy centers in diamond Phys Rev B 87014115

[19] Collins A T, Thomaz M F, Jorge M I B 1983 Luminescence decay time of the $1.945 \mathrm{eV}$ centre in type Ib diamond Journal of Physics C: Solid State Physics 162177

[20] Svelto O 1998 Principles of Lasers Springer

[21] Dréau A, Lesik M, Rondin L, Spinicelli P, Arcizet O, Roch J F, et al. 2011 Avoiding power broadening in optically detected magnetic resonance of single $\mathrm{NV}$ defects for enhanced dc magnetic field sensitivity Phys Rev B 84195204

[22] Clevenson H, Trusheim M E, Teale C, Schroder T, Braje D, Englund D 2015 Broadband magnetometry and temperature sensing with a light-trapping diamond waveguide Nat Phys 11 393-7

[23] Yeung T K, Sage D L, Pham L M, Stanwix P L, Walsworth R L 2012 Anti-reflection coating for nitrogen-vacancy optical measurements in diamond Applied Physics Letters 10025111

[24] Bar-Gill N, Pham L M, Jarmola A, Budker D, Walsworth R L 2013 Solid-state electronic spin coherence time approaching one second Nature Communications 41743

[25] McKnight L J, Dawson M D, Calvez S 2011 Diamond Raman Waveguide Lasers: Completely Analytical Design Optimization Incorporating Scattering Losses IEEE J Quantum Electron 47 1069- 